'Departamento de Nutrición, Facultad de Medicina,

Universidad de Chile. Santiago, Chile

2Programa para el Control de Tabaquismo, Organización Mundial de la Salud. aPhD.

${ }^{b}$ Nutricionista, alumna del Magíster en Salud Pública, Universidad de Chile.

Recibido el 30 de junio de 2017 , aceptado el 23 de enero de 2018.

Financiado por el Fondo Nacional de Desarrollo Científico y Tecnológico (Fondecyt) proyectos 1010572 y 1140453. Los autores no declaran conflictos de interés.

Correspondencia a: Dr. Hugo Amigo Independencia 1027. Santiago, Chile. hamigo@med.uchile.cl

\section{Patrón de tabaquismo y nivel socioeconómico en dos cohortes de adultos jóvenes}

\author{
HUGO AMIGO ${ }^{1, a}$, DANISSA ALE ${ }^{1}$, NICOL VARELA ${ }^{1, b}$, \\ ARMANDO PERUGA ${ }^{2, a}$, PATRICIA BUSTOS ${ }^{1}$
}

\section{Pattern of smoking and socioeconomic status in two cohorts of young adults}

\begin{abstract}
Background: One in five deaths that occur in Chile can be attributed to smoking whose prevalence remains high, despite interventions aimed at reducing it. Aim: To compare the prevalence of smoking and its intensity among young adults born 15 years apart and determine their association with socioeconomic status (SES). Material and Methods: Two cohorts of young adults living in the Valparaiso Region of Chile were evaluated in the third decade of life. Cohort 1 was evaluated between 2000 and $2002(n=1232)$ and cohort 2 between 2014 and 2017 ( $n=1078)$. Results: In cohort 1, 57.5\% (95\% Confidence Interval (CI) 54.6-58.7) of the subjects reported smoking, with a median of 3 (Interquartile range (ICR:1-6) cigarettes/day. This percentage fell to 40.2\% (CI: 37.5-43.1) with a similar median in cohort 2. Analyzing cohort 2, the odds ratio (OR) for smoking was 2.24 (CI 1.48-3.38) in the medium SES, compared with the medium high SES. The figures for low medium and low SES were 2.72 (CI: 1.85-3.99) and 3.01 (1.85-4.88). Similarly, in this cohort there was a significantly higher risk of being a heavy smoker in lower SES. No associations between smoking or its intensity and SES were observed in cohort 1. Conclusions: Smoking behavior has decreased among young adults evaluated at the same age in two generational cohorts in the third decade of life. In the most recent cohort analyzed, smoking and its intensity increase along with a decrease in SES.
\end{abstract}

(Rev Med Chile 2018; 146: 168-174)

Key words: Cohort Studies; Smoking; Young adult.
$\mathrm{E}$ 1 tabaco es responsable de aproximadamente 7 millones de muertes al año ${ }^{1}$, a pesar de ser una de las principales causas modificables de muerte y que cerca de una tercera parte de la población mundial está cubierta con alguna intervención destinada a reducir esta adicción. La persistencia de su importancia como factor de riesgo, indica que la efectividad de las medidas aún constituye un enorme desafío ${ }^{2,3}$.

A pesar de las evidencias sobre la importancia del efecto del tabaquismo en algunas enfermedades crónicas, la información en el país ha sido limitada ${ }^{4-6}$. La mayoría de los estudios se concentran en los efectos a largo plazo sobre enfermedades como cáncer pulmonar, enfermedad obstructiva pulmonar, infarto del miocardio y accidente vascular encefálico, entre otras ${ }^{7,8}$, así se ha mencionado que un tercio de todas las muertes por cáncer en países occidentales se atribuyen al tabaco y últimamente se ha señalado que la mitad de los infartos que ocurren en mujeres de mediana edad se deberían a esta causa9 .

La prevalencia del hábito tabáquico en los países del "cono sur" es una de las más altas a 
nivel del continente americano ${ }^{10}$ y los últimos datos nacionales chilenos indican que la mitad de los varones y alrededor de $40 \%$ de las mujeres han fumado el último mes aunque la intensidad del hábito es relativamente baja ${ }^{11}$. Estas cifras y sus repercusiones sobre la salud de las personas han apremiado acciones las cuales abarcan desde el ámbito individual para disminuir su consumo (por ejemplo el uso de publicidad mostrando los efectos dañinos del tabaco) hasta el nivel colectivo, con la creación de espacios libres del humo de tabaco y el aumento del impuesto a los cigarrillos ${ }^{12,13}$.

En conjunto estas medidas comienzan a mostrar sus logros. El informe sobre "carga global de enfermedades" ha destacado a Chile como uno de los 18 países con disminución del tabaquismo ${ }^{14}$. También la última encuesta de Calidad de Vida y Salud (ENCAVI), que entre 2000 y 2006 mostraba una prevalencia estancada en alrededor de $40 \%$ en los adultos, registra 30,1\% en 2015. Sin embargo, esta encuesta muestra que los adultos jóvenes comparativamente es el grupo más refractario, manteniendo una prevalencia que supera $40 \%{ }^{15}$.

Un desafío interesante es develar el papel que juega la posición socioeconómica en la disminución del hábito tabáquico. Este podría influir directamente por limitación, dado el aumento del precio, en cuyo caso podría esperarse mayor efecto en los estratos más bajos y en los más jóvenes o simplemente por la existencia de diferentes hábitos $^{16,17}$. También se ha mencionado que hay una tendencia en las mujeres, las cuales fuman más a medida que se incrementa su nivel socioeconómico, no demostrándose claramente lo mismo en varones ${ }^{18}$.

En vista de los antecedentes expuestos, el objetivo de este trabajo es comparar la prevalencia del tabaquismo y su intensidad en jóvenes adultos de las comunas de Limache y Olmué, Región de Valparaíso Chile, nacidos con 15 años de diferencia y constatar si estas características difieren según nivel socioeconómico (NSE). Estas comunas son semirurales con algo más de 61.000 habitantes y situadas a alrededor de $120 \mathrm{Km}$ de la capital del país, Santiago, Chile.

\section{Metodología}

El presente estudio se ha diseñado a partir de la información de dos cohortes no concurrentes: Cohorte $1(\mathrm{n}=1.232)$, cuyos miembros fueron seleccionados aleatoriamente de los nacidos entre 1974 y 1978 en el hospital de Limache y entrevistados entre 2000 y 2002 y Cohorte $2(n=1.078)$, que corresponde a una muestra de los nacidos entre 1988 y 1992, evaluados entre 2014 y 2017.

La recolección de datos incluyó una encuesta cuyas preguntas sobre tabaquismo han sido validadas internacionalmente ${ }^{19}$, la cual fue aplicada por personal capacitado. Se preguntó por el consumo actual de tabaco (al momento de la entrevista) y en caso que la respuesta fuera positiva, se preguntó por el número de cigarrillos diarios, considerando el consumo ocasional (fin de semana por ejemplo). El nivel socioeconómico se determinó mediante el nivel educacional y ocupación del jefe de hogar. Con estos datos se construyó una matriz basada en la propuesta para la Clasificación Socioeconómica Europea (Esomar) y que ha sido adaptada a la realidad chilena y validada mediante un estudio empírico $^{20}$. Esta metodología clasifica el nivel socioeconómico en cinco grupos: bajo, medio bajo, medio, medio alto y alto.

Para la caracterización de la muestra se hicieron análisis descriptivos, se determinó normalidad utilizando la prueba de Shapro Wilk y como la intensidad de consumo no sigue una distribución normal, se compararon las medianas, utilizándose la prueba $U$ de Mann Whitney para verificar significancia estadística. Para los datos categóricos se utilizó chi cuadrado. Para analizar la asociación entre NSE y fumar en el momento de la entrevista se efectuaron análisis de regresión logística binaria cuando la variable de tabaquismo fue la condición de fumador activo (sí/no), considerando como grupo de referencia a las personas clasificadas en el grupo socioeconómico medio alto y alto. Para analizar la asociación entre NSE e intensidad del consumo de cigarrillos se utilizó regresión logística ordinal clasificada en cuatro niveles ordenados, desde un valor mínimo a un máximo de 6 o más cigarrillos/día, niveles entre los que se distribuyeron los fumadores en proporciones semejantes, considerándose como grupo de referencia a los no fumadores y el NSE medio alto y alto. Los análisis consideraron el control de variables de confusión e interacción.

Para construir la base de datos se utilizó el programa Epi-data 3,1 a través de una digitación doble. Para los diferentes procedimientos estadísticos se utilizó el programa SPPS en su versión 22 y Stata versión 13. 
En ambas evaluaciones, los entrevistados firmaron un "Consentimiento Informado", en el cual se indicaba los procedimientos que se utilizarían además que la participación era voluntaria y que los datos se manejarían confidencialmente, respetando el anonimato. Este estudio contó con la aprobación del Comité de ética para Investigación en seres humanos de la Facultad de Medicina de la Universidad de Chile.

\section{Resultados}

La edad promedio de las cohortes fue similar $(\mathrm{p}=0,187)$, lo que es concordante con el diseño del estudio. En la cohorte 1, evaluada al inicio de la década de los 2000 la proporción de los que indicaban que eran fumadores alcanzó a más de la mitad de la muestra disminuyendo en 17,3 puntos porcentuales en la cohorte 2 , recientemente evaluada ( $\mathrm{p}<0,0001)$. El tabaquismo, siempre fue mayor en hombres, en la cohorte 1 alcanzó una diferencia de 16,8 puntos porcentuales, mientras que en la cohorte 2 fue de 8,9 puntos en relación al sexo femenino $(\mathrm{p}<0,001)$. La intensidad del tabaquismo (considerando solo los que fuman) medido en medianas, es similar entre las cohortes $(\mathrm{p}<0,631)$ siendo esta intensidad mayor en hombres $(\mathrm{p}<0,001)$. En relación al NSE se observa una mejoría de una cohorte a la otra, disminuyendo la proporción de los clasificados en NSE bajo en la cohorte 2 en 12,2 y aumentando en 14,9 puntos porcentuales las personas clasificadas en nivel socioeconómico medio alto y alto, como puede observarse en la Tabla 1.

Para verificar la asociación entre NSE y tabaquismo se elaboró un modelo de regresión logística binaria, en la que se constató una interacción significativa entre pertenecer a una determinada cohorte y NSE, comprobándose que en la cohorte 1 no se observan asociaciones significativas, con razones de disparidad (OR) cercanas a la unidad entre las distintas categorías socioeconómicas, mientras que en la cohorte 2 se observa un incremento progresivo del riesgo de fumar a medida que el nivel socioeconómico es más bajo, compro-

Tabla 1. Características generales de la muestra, según sexo

\begin{tabular}{|c|c|c|c|c|c|c|c|c|c|}
\hline \multirow[t]{2}{*}{ Variables } & \multicolumn{4}{|c|}{ Cohorte nacidos entre 1974-1978 } & \multicolumn{4}{|c|}{ Cohorte nacidos entre 1988-1992 } & \multirow[t]{2}{*}{ p value** } \\
\hline & Total & Hombres & Mujeres & $\begin{array}{c}\mathbf{p} \\
\text { value* }\end{array}$ & Total & Hombres & Mujeres & $\underset{\text { value* }}{\mathbf{p}}$ & \\
\hline Número (\%) & 1.232 & $\begin{array}{c}559 \\
(45,4)\end{array}$ & $\begin{array}{c}673 \\
(54,6)\end{array}$ & 0,001 & 1.078 & $\begin{array}{c}508 \\
(47,1)\end{array}$ & $\begin{array}{c}570 \\
(52,9)\end{array}$ & 0,05 & 0,670 \\
\hline $\begin{array}{l}\text { Edad (años) }{ }^{\dagger} \\
\text { Mediana (EIC) }\end{array}$ & $\begin{array}{c}25,0 \\
(24-26)\end{array}$ & $\begin{array}{c}25,0 \\
(24-26)\end{array}$ & $\begin{array}{c}25,0 \\
(23-25)\end{array}$ & 0,031 & $\begin{array}{c}24,7 \\
(23,6-26,0)\end{array}$ & $\begin{array}{c}24,7 \\
(23,7-26,0)\end{array}$ & $\begin{array}{c}24,7 \\
(23,6-26,1)\end{array}$ & 0,916 & 0,187 \\
\hline $\begin{array}{l}\text { Hábito tabáquico }{ }^{\ddagger} \\
\%(95 \% \mathrm{IC})\end{array}$ & $\begin{array}{c}57,5 \\
(54,6-58,7)\end{array}$ & $\begin{array}{c}66,7 \\
(62,8-70,6)\end{array}$ & $\begin{array}{c}49,9 \\
(46,2-53,7)\end{array}$ & $<0,001$ & $\begin{array}{c}40,2 \\
(37,3-43,1)\end{array}$ & $\begin{array}{c}45,2 \\
(42,3-48,2)\end{array}$ & $\begin{array}{c}36,3 \\
(33,4-39,2)\end{array}$ & 0,003 & 0,0001 \\
\hline $\begin{array}{l}\text { Intensidad hábito } \\
\text { tabáquico }{ }^{\dagger} \\
\text { Mediana (EIC) }\end{array}$ & $\begin{array}{c}3,00 \\
(1,0-6,0)\end{array}$ & $\begin{array}{c}4,00 \\
(1,0-8,0)\end{array}$ & $\begin{array}{c}3,00 \\
(1,0-5,0)\end{array}$ & $<0,001$ & $\begin{array}{c}3,0 \\
(2,0-7,0)\end{array}$ & $\begin{array}{c}3,0 \\
(3,0-10)\end{array}$ & $\begin{array}{c}3,0 \\
(1,0-6,0)\end{array}$ & 0,001 & 0,0001 \\
\hline \multicolumn{10}{|l|}{ NSE \% $(95 \% \text { IC })^{\ddagger}$} \\
\hline Bajo & $\begin{array}{c}24,4 \\
(19,6-29,2)\end{array}$ & $\begin{array}{c}17,5 \\
(10,0-25,0)\end{array}$ & $\begin{array}{c}30,2 \\
(23,9-36,5)\end{array}$ & & $\begin{array}{c}12,2 \\
(6,6-17,8)\end{array}$ & $\begin{array}{c}12,8 \\
(4,7-20,9)\end{array}$ & $\begin{array}{c}11,6 \\
(3,9-19,3)\end{array}$ & & \\
\hline Medio Bajo & $\begin{array}{c}38,1 \\
(33,7-42,5)\end{array}$ & $\begin{array}{c}51,4 \\
(45,6-57,1)\end{array}$ & $\begin{array}{c}27,0 \\
(20,6-33,4)\end{array}$ & & $\begin{array}{c}43,3 \\
(38,8-47,8)\end{array}$ & $\begin{array}{c}46,5 \\
(40,1-52,9)\end{array}$ & $\begin{array}{c}40,4 \\
(34,0-46,7)\end{array}$ & & \\
\hline Medio & $\begin{array}{c}34,6 \\
(30,3-38,9)\end{array}$ & $\begin{array}{c}28,6 \\
(21,6-35,6)\end{array}$ & $\begin{array}{c}39,5 \\
(33,6-45,4)\end{array}$ & & $\begin{array}{c}26,7 \\
(21,6-31,8)\end{array}$ & $\begin{array}{c}24,7 \\
(17,1-32,2)\end{array}$ & $\begin{array}{c}28,6 \\
(21,6-35,5)\end{array}$ & & 0,00 \\
\hline Medio Alto-alto & $\begin{array}{c}2,9 \\
(2,7-3,05)\end{array}$ & $\begin{array}{c}2,5 \\
(1,99-3,01)\end{array}$ & $\begin{array}{c}3,3 \\
(2,89-3,71)\end{array}$ & & $\begin{array}{c}17,8 \\
(12,4-23,2)\end{array}$ & $\begin{array}{c}16,0 \\
(9,0-23,9)\end{array}$ & $\begin{array}{c}19,4 \\
(12,0-26,8)\end{array}$ & & \\
\hline
\end{tabular}

EIC: Espacio inter cuartil, IC= intervalo de confianza. *Significancia entre hombres y mujeres. **Significancia entre cohortes. †U de Mann Whitney; ${ }^{\ddagger}$ Chi cuadrado. 
Tabla 2. Asociación entre nivel socioeconómico y hábito tabáquico

\begin{tabular}{|c|c|c|c|}
\hline Nivel socioeconómico & $\begin{array}{c}\text { Fuma actualmente } \\
\text { OR }\end{array}$ & $\mathbf{9 5 \%}$ intervalo de confianza & p value \\
\hline \multicolumn{4}{|l|}{ Cohorte 1 (1974-1978) } \\
\hline Bajo & 1,101 & $0,54-2,23$ & 0,78 \\
\hline Medio Bajo & 0,962 & $0,48-1,93$ & 0,91 \\
\hline Medio & 0,974 & $0,49-1,95$ & 0,94 \\
\hline \multicolumn{4}{|l|}{ Cohorte 2 (1988-1992) } \\
\hline Bajo & 3,007 & $1,85-4,88$ & 0,0001 \\
\hline Medio Bajo & 2,720 & $1,85-3,99$ & 0,0001 \\
\hline Medio & 2,238 & $1,48-3,38$ & 0,0001 \\
\hline
\end{tabular}

Controlado por sexo, edad. Grupo de referencia: Nivel socioeconómico medio alto y alto.

bándose una OR de 3 al comparar los fumadores de nivel bajo con los de NSE medio alto y alto (Tabla 2).

Al explorar la asociación entre el NSE e intensidad del tabaquismo, nuevamente se verifica una interacción significativa entre pertenecer a una determinada cohorte y NSE, por los que se expresan los resultados separados por cohorte. En la cohorte 1 no se observan mayores asociaciones en ningunas de las categorías de intensidad del tabaquismo, mientras que en la cohorte 2 en todas las categorías de intensidad se observan OR significativos, mayores en el NSE bajo, teniendo como referencia el NSE medio y alto. En la categoría de mayor intensidad del NSE bajo llegan a OR de 4,73 $(\mathrm{p}<0,001)$ (Tabla 3$)$.

Tabla 3. Asociación entre nivel socioeconómico e intensidad del tabaquismo

\begin{tabular}{|c|c|c|c|c|}
\hline n de cigarros día & Nivel socioeconómico & OR & $\mathbf{9 5 \%}$ intervalo de Confianza & p value \\
\hline \multicolumn{5}{|c|}{ Cohorte 1 (nacidos entre 1974-1978) } \\
\hline 0,1 a 2.9 & $\begin{array}{l}\text { Bajo } \\
\text { Medio bajo } \\
\text { Medio }\end{array}$ & $\begin{array}{l}0,98 \\
1,13 \\
1,18\end{array}$ & $\begin{array}{l}0,39-2,42 \\
0,46-2,76 \\
0,48-2,87\end{array}$ & $\begin{array}{l}0,956 \\
0,783 \\
0,72\end{array}$ \\
\hline 3 a 5,9 & $\begin{array}{l}\text { Bajo } \\
\text { Medio bajo } \\
\text { Medio }\end{array}$ & $\begin{array}{l}1,48 \\
1,32 \\
1,22\end{array}$ & $\begin{array}{l}0,55-3,98 \\
0,50-3,51 \\
0,46-3,26\end{array}$ & $\begin{array}{l}0,44 \\
0,58 \\
0,69\end{array}$ \\
\hline Mayor de 6 & $\begin{array}{l}\text { Bajo } \\
\text { Medio bajo } \\
\text { Medio }\end{array}$ & $\begin{array}{l}0,63 \\
0,95 \\
0,54\end{array}$ & $\begin{array}{l}0,24-1,67 \\
0,37-2,43 \\
0,21-1,39\end{array}$ & $\begin{array}{l}0,357 \\
0,921 \\
0,200\end{array}$ \\
\hline \multicolumn{5}{|c|}{ Cohorte 2 (nacidos entre 1988-1992) } \\
\hline 0,1 a 2.9 & $\begin{array}{l}\text { Bajo } \\
\text { Medio bajo } \\
\text { Medio }\end{array}$ & $\begin{array}{l}3,31 \\
2,35 \\
2,00\end{array}$ & $\begin{array}{l}1,71-6,41 \\
1,35-4,07 \\
1,11-3,61\end{array}$ & $\begin{array}{l}0,001 \\
0,002 \\
0,021\end{array}$ \\
\hline 3 a 5,9 & $\begin{array}{l}\text { Bajo } \\
\text { Medio bajo } \\
\text { Medio }\end{array}$ & $\begin{array}{l}2,90 \\
2,22 \\
1,79\end{array}$ & $\begin{array}{l}1,42-5,89 \\
1,24-3,98 \\
0,95-3,36\end{array}$ & $\begin{array}{l}0,003 \\
0,007 \\
0,069\end{array}$ \\
\hline Mayor de 6 & $\begin{array}{l}\text { Bajo } \\
\text { Medio bajo } \\
\text { Medio }\end{array}$ & $\begin{array}{l}4,73 \\
4,29 \\
2,93\end{array}$ & $\begin{array}{l}1,84-12,1 \\
1,90-9,66 \\
1,23-6,96\end{array}$ & $\begin{array}{l}0,001 \\
0,001 \\
0,015\end{array}$ \\
\hline
\end{tabular}

Controlado por sexo y edad. Grupo de referencia socioeconómico: Nivel medio alto y altoGrupo de referencia de intensidad de tabaquismo: No fumar 


\section{Discusión}

En este estudio se comprueba que la prevalencia de tabaquismo ha disminuido en la población de adultos jóvenes que habitan en dos comunas de la Región de Valparaíso y que fueron evaluados a la misma edad y con los mismos instrumentos, en la tercera década de la vida. A su vez, solo en la última cohorte (evaluada recientemente) el tabaquismo se concentra en los NSE más bajos de la población al comparar con los de niveles medio alto y alto. Similar situación se constata en la intensidad del tabaquismo, observándose también que los pertenecientes a la última cohorte y clasificados en niveles socioeconómicos más bajos son los que más fuman (evaluado a través del número de cigarrillos fumados al día).

Esta situación puede deberse a mejorías de las condiciones socioeconómicas en la cual el tabaquismo habría disminuido en los niveles más altos y/o que esta disminución sea producto de las políticas destinadas a disminuir el tabaquismo, aunque esta posibilidad no puede afirmarse porque en la comparación de estas cohortes no se consideraron factores específicos relacionados con las campañas destinadas a desincentivar esta adicción.

A pesar de la disminución del tabaquismo, la frecuencia observada en los adultos jóvenes de este estudio es alta lo que comprueba que esta edad constituye uno de los grupos más adherentes a este hábito y confirma que en Chile continúan prevalencias altas en comparación con el resto de los países de América Latina ${ }^{21}$. Esta situación -como se mencionó previamente- ha llevado a que en el país se hayan efectuado campañas educativas y promulgado leyes destinadas a la disminución de este consumo.

La mayor prevalencia de tabaquismo en los niveles socioeconómicos bajos ha sido comunicada en otros países ${ }^{22,23}$, en este trabajo también se observa este comportamiento pero solo en la cohorte 2 verificándose en ella un efecto dosis respuesta o sea mientras mejora el NSE, menor es el riesgo de tabaquismo, este es un elemento que debiera ser considerado en la elaboración de programas destinados a disminuirlo.

La diferencia entre géneros ha sido documentada en la literatura ${ }^{16,24}$. El que sean los varones los que tengan mayor prevalencia de tabaquismo (en ambos períodos) confirma que son ellos el grupo a tener en consideración en la elaboración de programas anti tabaco. Los niveles observados en el sexo femenino son una alerta ya que en ellas se suman a este riesgo, otros ya identificados (por ejemplo el que tengan mayor IMC) o que la letalidad por algunas enfermedades crónicas (como el infarto del miocardio) sean mayores en este $\operatorname{sexo}^{25,26}$. Esto también es una amenaza, ya que en países desarrollados se ha observado un aumento del tabaquismo en mujeres y es posible que esta situación se extienda a países de menor desarrollo ${ }^{16}$. Este trabajo tiene la fortaleza de haber utilizado cuestionarios probados internacionalmente y aplicados por personal capacitado con experiencia en realizar este tipo de encuestas poblacionales y que el trabajo fue efectuado en la misma edad en dos cohortes que nacieron con un intervalo aproximado de 15 años y que habitan en dos comunas semi-rurales de la Región de Valparaíso. Como debilidad se puede indicar que las poblaciones difieren de su condición socioeconómica, siendo que en la cohorte más reciente se evidencian mejorías socioeconómicas a las cuales se les podría atribuir esta disminución del tabaquismo y no necesariamente puede ser explicado por el efecto de programas destinados a disminuir el consumo de cigarrillos. La mejoría del NSE observada en este estudio es concordante con lo reportado en el país, lo que se demuestra por el incremento del PNB per cápita y la disminución de la pobreza en el período transcurrido entre las dos evaluaciones $^{27,28}$. Se debe mencionar que para controlar posibles desbalances por sexo en la muestra, los análisis multivariados fueron controlados por el efecto de esta variable.

De este trabajo se pueden derivar antecedentes para la elaboración de programas destinados a reducir el consumo de tabaco, a pesar de la disminución observada en la frecuencia de consumo, los que deben considerar las condiciones socioeconómicas, focalizándose en personas de menores recursos e iniciarse tempranamente en la vida, como se ha realizado en otros lugares ${ }^{2,29}$. El grupo de los adultos jóvenes debe ser considerado como grupo prioritario y las campañas educativas deberían fortalecerse y ser más didácticas y finalmente, en el contenido de los programas debe considerarse la desigualdad socioeconómica y un enfoque de género.

Agradecimientos: al Dr. Claudio Vargas y 
Dr. Sergio Muñoz por su contribución en la estrategia de análisis de los datos y a la Dra. Paulina Pino por su revisión de la versión final del manuscrito.

\section{Referencias}

1. GBD 2015 Tobacco Collaborators. Smoking prevalence and attributable disease burden in 195 countries and territories, 1990-2015: a systematic analysis from the Global Burden of Disease Study 2015. Lancet (London, England). 2017; 389 (10082): 1885-906.

2. Hill S, Amos A, Clifford D, Platt S. Impact of tobacco control interventions on socioeconomic inequalities in smoking: review of the evidence. Tob Control 2014; 23 (e2): e89-97.

3. Purcell KR, O'Rourke K, Rivis M. Tobacco control approaches and inequity-how far have we come and where are we going? Health Promot Int 2015; 30 Suppl 2: ii89-101.

4. Pichón Riviere A, Bardach A, Caporale J, Alcaraz A, Augustovskin F, Caccavo F, Vallejos V, et al. Carga de Enfermedad atribuible al Tabaquismo en Chile 2014. Disponible en: www.iecs.org.ar. [Consultado el 22 de junio de 2017].

5. Medina E, Kaempffer AM, Cornejo E, Hernández E. [Smoking in Santiago, 1993-94]. Rev Med Chile 1995; 123 (5): 652-8.

6. Jadue L, Vega J, Escobar MC, Delgado I, Garrido C, Lastra $\mathrm{P}$, et al. [Risk factors for non communicable diseases: methods and global results of the CARMEN program basal survey]. Rev Med Chile 1999; 127 (8): 1004-13.

7. Ezzati M, López AD. Estimates of global mortality attributable to smoking in 2000. Lancet 2003; 362 (9387): 847-52.

8. Amigo H, Erazo M, Oyarzún M, Bello S, Peruga A. [Smoking and chronic obstructive pulmonary disease: attributable risk determination]. Rev Med Chile 2006; 134 (10): 1275-82.

9. Janzon E, Hedblad B, Berglund G, Engstrom G. Tobacco and myocardial infarction in middle-aged women: a study of factors modifying the risk. J Intern Med 2004; 256 (2): 111-8.

10. Menezes AM, López MV, Hallal PC, Muino A, Pérez-Padilla R, Jardim JR, et al. Prevalence of smoking and incidence of initiation in the Latin American adult population: the PLATINO study. BMC Public Health 2009; 9: 151: 1-8.

11. Ministerio de Salud de Chile. Encuesta Nacional de Salud, 2009-2010. Disponible en: http://web.minsal.cl/ portal/url/item/bcb03d7bc28b64dfe040010165012d23. pdf2010. [Consultado el 22 de junio de 2017].

12. Ministerio de Salud de Chile. Modifica Ley No 19.419, en materia de ambientes libres de humo de tabaco 2013. Disponible en: https://www.leychile.cl/Navegar?idNor$\mathrm{ma}=1047848$. [Consultado el 22 de junio de 2017].

13. Fundación Educación Popular en Salud. Chile Libre de Tabaco 2017. Disponible en: http://www.chilelibredetabaco.cl/category/campanas/impuesto-tabaco- campanas/ [Consultado el 22 de junio de 2017].

14. Institute for Health Metrics and Evaluation. Global Burden Disease. Disponible en: http://www.healthdata. org/gbd [Consultado el 25 de junio de 2017].

15. Ministerio de Salud de Chile. Encuesta de Calidad de Vida. Disponible en: http://web.minsal.cl/encuestade-calidad-de-vida-y-salud-del-minsal-674-de-la-poblacion-reconoce-tener-una-calidad-de-vida-buena-omuy-buena/ [Consultado el 25 de junio de 2017].

16. Huisman M, Kunst AE, Mackenbach JP. Educational inequalities in smoking among men and women aged 16 years and older in 11 European countries. Tob Control 2005; 14 (2): 106-13.

17. Ho LM, Schafferer C, Lee JM, Yeh CY, Hsieh CJ. The effect of cigarette price increases on cigarette consumption, tax revenue, and smoking-related death in Africa from 1999 to 2013. Int J Public Health 2017; 62 (8): 899-909.

18. Amos A, Greaves L, Nichter M, Bloch M. Women and tobacco: a call for including gender in tobacco control research, policy and practice. Tob Control 2012; 21 (2): 236-43.

19. Organización Mundial de la Salud Serie de preguntas básicas de la Encuesta Mundial de Tabaquismo en adultos, disponible en http: www.who.int/tobacco/ publication/surveillance/tqs_es_final.pdf. (Consultado 16 de octubre de 2017).

20. Adimark. El Nivel Socio Económico Esomar. Manual de Aplicación, 2000 http://www.microweb.cl/idm/documentos/ESOMAR.pdf (consultado el 25 de junio de 2017).

21. Bardach A, Perdomo HA, Gandara RA, Ciapponi A. [Income and smoking prevalence in Latin America: a systematic review and meta-analysis]. Rev Panam Salud Publica 2016; 40 (4): 263-71.

22. Peretti-Watel P, L'Haridon O, Seror V. Time preferences, socioeconomic status and smokers' behaviour, attitudes and risk awareness. Eur J Public Health 2013; 23 (5): 783-8.

23. Huisman M, Van Lenthe FJ, Giskes K, Kamphuis CB, Brug J, Mackenbach JP. Explaining socio-economic inequalities in daily smoking: a social-ecological approach. Eur J Public Health 2012; 22 (2): 238-43. 
24. Bilal U, Beltrán P, Fernández E, Navas-Acien A, Bolumar F, Franco M. Gender equality and smoking: a theory-driven approach to smoking gender differences in Spain. Tob Control 2016; 25 (3): 295-300.

25. Ministerio de Salud de Chile. Encuesta Nacional de Consumo Alimetario. ENCA 2014. Disponible en: http://web.minsal.cl [Consultado el 22 de junio de 2017].

26. Thomas D. [Cardiovascular risk of smoking by gender]. Presse Med 2017; 46 (7-8): 681-7.

27. Ministerio de Desarrollo Social de Chile. Encuesta de caracterización socioeconómica nacional. CASEN 2015. Disponible en: htpp:/www.oecd.org/eco/growth/Chile. pdf [Consultado el 21 de junio de 2017].

28. Trading Economics. Gross domestic product GDP per capita US current price 2014. Disponible en: http:// www.tradingeconomics.com [Consultado el 22 de junio de 2017].

29. Brown T, Platt S, Amos A. Equity impact of European individual-level smoking cessation interventions to reduce smoking in adults: a systematic review. Eur J Public Health 2014; 24 (4): 551-6. 